\title{
Exercise cardiovascular magnetic resonance reveals reduced cardiac reserve in pediatric cancer survivors with impaired cardiopulmonary fitness
}

Stephen Foulkes ${ }^{1,2}$, Benedict T. Costello ${ }^{1,3}$, Erin J. Howden ${ }^{1}$, Kristel Janssens ${ }^{1}$, Hayley Dillon ${ }^{1,2}$, Claudia Toro ${ }^{4}$, Piet Claus ${ }^{5}$, Steve F. Fraser ${ }^{2}$, Robin M. Daly ${ }^{2}$, David A. Elliott ${ }^{6,4,7}$, Rachel Conyers ${ }^{6,4,8+}$ and Andre La Gerche ${ }^{1,3^{*+}}$ (D)

\begin{abstract}
Background: Pediatric cancer survivors are at increased risk of cardiac dysfunction and heart failure. Reduced peak oxygen consumption (peak $\mathrm{VO}_{2}$ ) is associated with impaired cardiac reserve (defined as the increase in cardiac function from rest to peak exercise) and heart failure risk, but it is unclear whether this relationship exists in pediatric cancer survivors. This study sought to investigate the presence of reduced peak $\mathrm{VO}_{2}$ in pediatric cancer survivors with increased risk of heart failure, and to assess its relationship with resting cardiac function and cardiac haemodynamics and systolic function during exercise.

Methods: Twenty pediatric cancer survivors (8-24 years; 10 male) treated with anthracycline chemotherapy \pm radiation underwent cardiopulmonary exercise testing to quantify peak $\mathrm{VO}_{2}$, with a value $<85 \%$ of predicted defined as impaired peak $\mathrm{VO}_{2}$. Resting cardiac function was assessed using 2- and 3-dimensional echocardiography, with cardiac reserve quantified from resting and peak exercise heart rate, stroke volume index (SVI) and cardiac index (Cl) using exercise cardiovascular magnetic resonance (CMR).

Results: Twelve of 20 survivors (60\%) had reduced peak $\mathrm{VO}_{2}$ (70 $\pm 16 \%$ vs. $97 \pm 14 \%$ of age and gender predicted). There were no differences in echocardiographic or CMR measurements of resting cardiac function between survivors with normal or impaired peak $\mathrm{VO}_{2}$. However, those with reduced peak $\mathrm{VO}_{2}$ had diminished cardiac reserve, with a lesser increase in $\mathrm{Cl}$ and SVI during exercise (Interaction $P<0.01$ for both), whilst the heart rate response was similar $(P=0.71)$.

Conclusions: Whilst exercise intolerance is common among pediatric cancer survivors, it is poorly explained by resting measures of cardiac function. In contrast, impaired exercise capacity is associated with impaired haemodynamics and systolic functional reserve measured during exercise. Consequently, measures of cardiopulmonary fitness and cardiac reserve may aid in early identification of survivors with heightened risk of long-term heart failure.
\end{abstract}

Keywords: Cardiotoxicity, Cardiac function, Cardiopulmonary fitness, Cancer, Exercise CMR

\footnotetext{
* Correspondence: Andre.LaGerche@baker.edu.au

${ }^{\dagger}$ Rachel Conyers and Andre La Gerche contributed equally to this work.

'Department of Sports Cardiology, Baker Heart and Diabetes Institute, 75 Commercial Rd, Melbourne, VIC 3004, Australia

${ }^{3}$ Cardiology Department, St Vincent's Hospital Melbourne, Melbourne, VIC, Australia

Full list of author information is available at the end of the article
}

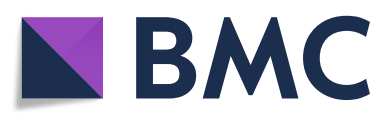

(- The Author(s). 2020 Open Access This article is licensed under a Creative Commons Attribution 4.0 International License, which permits use, sharing, adaptation, distribution and reproduction in any medium or format, as long as you give appropriate credit to the original author(s) and the source, provide a link to the Creative Commons licence, and indicate if changes were made. The images or other third party material in this article are included in the article's Creative Commons licence, unless indicated otherwise in a credit line to the material. If material is not included in the article's Creative Commons licence and your intended use is not permitted by statutory regulation or exceeds the permitted use, you will need to obtain permission directly from the copyright holder. To view a copy of this licence, visit http://creativecommons.org/licenses/by/4.0/. The Creative Commons Public Domain Dedication waiver (http://creativecommons.org/publicdomain/zero/1.0/) applies to the data made available in this article, unless otherwise stated in a credit line to the data. 


\section{Background}

Current 5-year survival rates for pediatric malignancies in developed nations have increased from 58\% in 19751977 to $83 \%$ for those diagnosed between 2005 and 2011 [1]. However, these dramatic improvements in cancerrelated survival have accentuated the long-term consequences of cancer treatment, with survivors of pediatric malignancies experiencing increased morbidity and early mortality due to treatment-related chronic health conditions [2-4]. Cardiovascular disease is the most common morbidity experienced by pediatric cancer survivors with $11 \%$ of survivors having a diagnosable cardiac condition prior to 40 years of age, of which heart failure is the predominant cause [5]. This has led to a shift in focus, whereby treatment success is determined by the balance between treatment efficacy and the risk of treatmentrelated toxicities [6]. However, despite international recommendations, less than $28 \%$ of survivors are receiving appropriate cardiac screening [7].

The current paradigm for preventing cardiotoxicity relies on the early detection of cardiac dysfunction when it may be more amenable to initiation of heart failure therapy [8]. Clinical decision making is based on serial changes in resting left ventricular (LV) ejection fraction (LVEF) or LV fractional shortening (FS), which is assessed using two-dimensional (2D) echocardiography or radionucleotide ventriculography [8]. The identification of LVEF or LV FS below the lower limit of normal should prompt the initiation of cardioprotective heart failure therapies $[6,8]$. For those patients who are undergoing active treatment, this may also result in treatment modification, delay or cessation [6]. However, clinical decision making is complicated by the lack of sensitivity of LVEF and LV FS in explaining treatmentinduced functional limitations, patients' symptoms and longer-term heart failure events $[9,10]$. This has resulted in increased interest in alternative surveillance methods for identifying cardiac dysfunction and quantifying heart failure risk [8]. The assessment of myocardial deformation via novel resting echocardiographic or cardiovascular magnetic resonance (CMR) methods are emerging as more sensitive markers of sub-clinical cardiac dysfunction in various cancer groups. However, the degree to which these measures are associated with functional impairment and quantifiable heart failure symptoms such as exercise intolerance is unclear.

Exercise intolerance, defined as reduced cardiopulmonary fitness or peak oxygen consumption (peak $\mathrm{VO}_{2}$ ) measured from a cardiopulmonary exercise test (CPET), has proven clinical utility in quantifying heart failure symptoms and predicting prognosis $[11,12]$. Recent advances in cardiac imaging have enabled the measurement of cardiac function augmentation with exercise (termed "cardiac reserve") to be assessed with greater accuracy and have been shown to be strongly associated with peak oxygen consumption $\left(\mathrm{VO}_{2}\right)$ [13]. Thus, both peak $\mathrm{VO}_{2}$ and cardiac reserve may provide greater sensitivity for detecting sub-clinical cardiac dysfunction than resting measures [12]. However, associations between exercise intolerance and cardiac function (including cardiac reserve) in pediatric cancer survivors remain inconclusive $[14,15]$.

Therefore, the aim of this study was to explore the presence of exercise intolerance in survivors of childhood and adolescent malignancy at high risk of cardiac dysfunction, and to explore its relationship with measures of resting cardiac function, and exercise-based measures of cardiac haemodynamics and systolic function.

\section{Methods}

Participant population and study design

Participants were cancer survivors treated or undergoing active treatment for pediatric haematologic malignancy at the Royal Children's Hospital, Monash Medical Centre or Alfred Hospital, Melbourne. Participation involved recruitment from attendance at routine outpatient clinics, with participants providing informed written consent prior to participation in the study. This study was approved by the ethics committee of the Melbourne Royal Children's Hospital (HREC 35102D) and the Alfred Hospital (HREC 00315) and was conducted in accordance with the Declaration of Helsinki guidelines.

Enrollment criteria included: (1) previous treatment with anthracycline chemotherapy with or without craniospinal or chest-targeted radiotherapy, and (2) height > $120 \mathrm{~cm}$ (in order to reach pedals for exercise testing). Participants were excluded if they had a previous history of severe symptomatic cardiac disease or had a contraindication to CMR.

\section{Outcome measures}

Comprehensive resting and exercise evaluation was completed at the Baker Heart and Diabetes Institute, Melbourne. Participants were evaluated with i) comprehensive resting transthoracic echocardiogram to assess LVEF, LV FS and global longitudinal strain (GLS), ii) a maximal CPET to evaluate peak $\mathrm{VO}_{2}$ as an indication of cardiopulmonary fitness, and iii) resting and exercise CMR (exCMR) to determine resting GLS, and resting and peak exercise LVEF, right ventricular (RV) ejection fraction (RVEF), stroke volume index (SVI), heart rate and cardiac index $(\mathrm{CI})$ as measures of cardiac reserve. Participants were classified by normal peak $\mathrm{VO}_{2}$ or impaired peak $\mathrm{VO}_{2}$ defined as peak $\mathrm{VO}_{2} \geq 85 \%$ agepredicted values. Cardiovascular risk factors were assessed at the time of study enrollment. Participants were assessed for hypertension, diabetes mellitus, chronic renal insufficiency and body mass index (BMI). 
Haemoglobin concentration was obtained from routine clinical blood results conducted within 2 months of the study visit, and participants were also screened for pulmonary comorbidities that could provide an alternative explanation for exertional intolerance.

\section{Exercise capacity}

CPET was conducted on an electronically braked cycle ergometer (Lode Excalibur, Groningen, The Netherlands) for the measurement of peak $\mathrm{VO}_{2}$. Workload was increased using a ramp protocol which began at 10-25 watts (W) and progressively increased at 10$30 \mathrm{~W} / \mathrm{min}$ until volitional exhaustion. Respiratory gas analysis was measured continuously throughout the test using a calibrated metabolic cart (True One 2400, Parvomedics, Salt Lake City, Utah, USA). Heart rate, rhythm and presence of ischemia was measured continuously by 12-lead electrocardiogram (ECG) (Norav Medical, Wiesbaden, Germany). Peak $\mathrm{VO}_{2}$ was defined as a 30-s rolling average of the six highest 5 -s oxygen uptake values. Participants were classified as having impaired peak $\mathrm{VO}_{2}$ if they achieved $<85 \%$ of predicted values for children/ adolescents [16] or adults [17] in line with American Thoracic Society guidelines for CPET interpretation [18]. The anaerobic threshold was calculated using the V-slope method [19], with the $\mathrm{VO}_{2}$ at the anaerobic threshold expressed as an absolute value and as a proportion of each participant's predicted peak $\mathrm{VO}_{2}$.

\section{Echocardiography}

Resting cardiac function was assessed from a comprehensive resting echocardiogram (Vivid E95, General Electric Healthcare, Milwaukee, Wisconsin, USA), with images saved in a digital format for offline analysis (Echopac v13.0.00, General Electric Healthcare). A full-volume threedimensional data set was acquired. LV end-diastolic and end-systolic volumes were measured according to standard recommendations [20]. Two-dimensional GLS was quantified from three apical views at a temporal resolution of 60 $90 \mathrm{frame} / \mathrm{s}$. The average negative value on the strain curve was reported as GLS. Doppler measures of diastolic function were acquired and analyzed per guideline recommendations [21].

\section{CMR imaging}

CMR imaging was performed with a $3 \mathrm{~T}$ CMR system (MAGNETOM Prisma, Siemens Healthineers, Erlangen, Germany) with a 5-element phased array coil. Ungated real-time balanced steady state free-precision cine imaging was performed with a parallel imaging acceleration factor of 3 and subsequent GRAPPA reconstruction without cardiac or respiratory gating. Forty (during exercise) or 100 (at rest) consecutive frames were acquired with a temporal resolution of $39 \pm 3 \mathrm{msec}$ in a short- and long-axis plane. Typical imaging parameters were field of view $=360 \mathrm{~mm}, 128 \times 128$ matrix, voxel size $=2.8 \times$ $2.8 \times 8 \mathrm{~mm}$, slice gap $=0 \mathrm{~mm}$, echo time $=1.18 \mathrm{~ms}$, echo spacing $=2.6 \mathrm{~ms}$, flip angle $=48^{\circ}-68^{\circ}$, bandwidth $=1260$ $\mathrm{Hz} / \mathrm{Px}$.

Resting global longitudinal strain GLS was calculated using feature tracking on the cine images (CVI42, Circle Cardiovascular Imaging Inc. Calgary, Alberta, Canada). The myocardium was defined according to American Heart Association segments by placing a marker across the mitral valve annulus and from the annulus to the apex on long axis images, and by marking endocardial and epicardial borders in the short axis volumetric stack and three apical cine images (4 chamber, 2 chamber, 3 chamber). Markers were placed at both RV insertion points on the short axis images. The feature-tracking algorithm within the CVI42 software calculated GLS.

Exercise CMR imaging The biventricular response to exercise was measured using a real-time CMR protocol that has been described in detail previously and validated against invasive measures [13]. Images were obtained at rest, and whilst cycling using a CMR compatible ergometer (MR Ergometer Pedal, Lode, Groningen, Netherlands) at an intensity equal to $60 \%$ of maximal power output obtained during the upright incremental cycle exercise test (demonstrated in an additional movie file - Additional file 1) which we have previously shown approximates near maximal exercise in a supine position [13]. Exercise was maintained for up to $3 \mathrm{~min} ; 30 \mathrm{~s}$ to achieve a physiological steady-state and approximately 1 to $2.5 \mathrm{~min}$ for image acquisition.

Images were analyzed on a software program developed in-house (RightVol - Right Volume Leuven, Leuven, Belgium) with retrospective respiratory and ECG gating to ensure endocardial contouring could be performed at the same point in the respiratory cycle. LV and RV endocardial contours were then manually traced on the short axis image with simultaneous reference to the horizontal long axis plane, thereby enabling constant referencing of the atrioventricular valve plane. Ventricular volumes were calculated by a summation of disks, with trabeculations and papillary muscle considered part of the ventricular blood pools. SVI was measured as the difference between enddiastolic volume and end-systolic volumes indexed to body surface area, while CI was calculated as (RV SV $+\mathrm{LV}$ $\mathrm{SV} / 2) \times$ heart rate $(\mathrm{HR})$ indexed to body surface area. Using this technique, our group has demonstrated excellent inter-observer $(R=0.98$ and $R=0.97$ for $L V$ and $R V$ SV, respectively) and inter-study reproducibility $(R=0.98$ for cardiac output) [13]. Peripheral muscle arterio-venous oxygen difference (a- $\mathrm{vO}_{2}$ diff) was calculated indirectly according to the Fick principle [22], using peak $\mathrm{VO}_{2}$ from 
the CPET and peak cardiac output measured by exercise CMR.

\section{Statistical analysis}

Data analyses were conducted using SPSS statistical software (version 24, Statistical Package for the Social Sciences, International Business Machines, Inc., Armonk, New York, USA). The distribution of continuous variables was tested using a Kolmogorov-Smirnov test. Categorical variables are expressed as $\mathrm{n}$ (\%), whilst continuous variables are expressed as mean \pm standard deviation or median (interquartile range) as appropriate. Differences in participant characteristics were compared by independent $\mathrm{t}$-tests or Mann-Whitney $\mathrm{U}$ tests for continuous variables, and chi-square or fisher's exact tests for categorical variables. Differences in the exCMR cardiac response to exercise was assessed using repeated measures ANOVA (with an interaction term for group $\mathrm{x}$ exercise response), with post-hoc analysis conducted using Bonferroni correction. Pearson correlations were used to test for associations between peak $\mathrm{VO}_{2}$ and measures of cardiac function and peripheral muscle oxygen extraction. A 2-sided $P$ value of $<0.05$ was considered statistically significant.

\section{Results}

\section{Participant characteristics}

A total of 20 patients participated in the study, and the summary of participant demographics and characteristics are summarized in Table 1. The median age of participants was 17 years (range 8-24), of which 10 were male $(50 \%)$. The most common tumor type was acute lymphoblastic leukemia $(n=10,50 \%)$. The median anthracycline dose was $175 \mathrm{mg} / \mathrm{m}^{2}$ with most participants $(n=16)$ receiving between 150 and $350 \mathrm{mg} / \mathrm{m}^{2}$ and two participants exceeding $450 \mathrm{mg} / \mathrm{m}^{2}$. Six participants had radiation together with chemotherapy, of which two received total body radiation, two received cranio-spinal radiation, and two received field radiotherapy to the chest. Two survivors had previously undergone allogeneic stem cell transplantation two years previously. There were no pulmonary comorbidities in the study cohort, however seven participants (35\%) had at least one cardiovascular disease risk factor. Both groups had a similar BMI (Normal: $21.7 \pm 4.1 \mathrm{~kg} / \mathrm{m}^{2}$ vs Impaired: $20.4 \pm 4.0$ $\mathrm{kg} / \mathrm{m}^{2}, P=0.45$ ) and haemoglobin concentration (Normal: $135 \mathrm{~g} / \mathrm{L}$ [104, 184] vs Impaired: $128 \mathrm{~g} / \mathrm{L}$ [93, 144], $P=0.18$ ). However, the impaired fitness group tended to have a higher proportion of females $(67 \%$ vs $25 \%, P=$ $0.085)$ and tended to have a shorter time from initial diagnosis (median 2.6 vs 5.1 years, $P=0.076$ ). There were no other significant differences in demographic, diagnosis or treatment characteristics between groups.
Table 1 Demographic and treatment characteristics $(n=20)$

\begin{tabular}{|c|c|}
\hline Current age (years) & $17(8-24)$ \\
\hline Age at diagnosis (years) & $13(6-21)$ \\
\hline Time since diagnosis (years) & $4.4(0.5-15.3)$ \\
\hline Peak $\mathrm{VO}_{2}(\mathrm{~L} / \mathrm{min})$ & $1.9 \pm 0.9$ \\
\hline$\%$ Predicted peak $\mathrm{VO}_{2}(\%)$ & $81 \pm 20$ \\
\hline Haemoglobin, g/L & $129(93,184)$ \\
\hline \multicolumn{2}{|l|}{ Sex } \\
\hline Male & $10(50 \%)$ \\
\hline Female & $10(50 \%)$ \\
\hline \multicolumn{2}{|l|}{ Diagnosis } \\
\hline Acute lymphoblastic leukaemia & $12(60 \%)$ \\
\hline Acute myeloid leukaemia & $5(25 \%)$ \\
\hline Hodgkin rumour & $2(10 \%)$ \\
\hline Wilms rumour & $1(5 \%)$ \\
\hline \multicolumn{2}{|l|}{ Treatment Details } \\
\hline Anthracycline, cumulative dose exposure $\left(\mathrm{mg} / \mathrm{m}^{2}\right)$ & $175(89-670)$ \\
\hline Chest-targeted radiation & $2(10 \%)$ \\
\hline Craniospinal radiation & $2(10 \%)$ \\
\hline Total body radiation & $2(10 \%)$ \\
\hline Allogeneic SCT & $2(10 \%)$ \\
\hline \multicolumn{2}{|l|}{ Cardiovascular risk factors } \\
\hline Hypertension & $1(5 \%)$ \\
\hline Diabetes & $2(10 \%)$ \\
\hline Dyslipidaemia & $3(15 \%)$ \\
\hline Chronic renal insufficiency & $1(5 \%)$ \\
\hline $\mathrm{BMI}>25 \mathrm{~kg} / \mathrm{m}^{2}$ & $3(15 \%)$ \\
\hline
\end{tabular}

Values are mean $\pm S D$, median (range) or $\mathrm{n}(\%)$. Abbreviations: SCT Stem cell transplantation, BMI Body mass index

\section{Exercise capacity}

Differences in exercise capacity and CPET parameters for those with normal $(n=8)$ and impaired peak $\mathrm{VO}_{2}$ $(n=12)$ are summarized in Table 2 . All subjects reached $>85 \%$ predicted maximal heart rate, with all but one subject obtaining a peak respiratory exchange ratio $>1.1$ indicative of exercise to maximal or near-maximal intensity. Survivors with impaired fitness achieved a peak $\mathrm{VO}_{2}$ that was on average $30 \%$ below predicted values, compared to $3 \%$ below predicted for survivors with normal peak $\mathrm{VO}_{2}$. Survivors with impaired fitness achieved a significantly lower absolute $(P=0.018)$ and bodyweight indexed peak $\mathrm{VO}_{2}(P=0.003)$ to those with normal fitness, whilst also tending to achieve a lower peak workload $(P=0.089)$. In contrast, the heart rate and ventilatory response was similar $(P>0.05$ for all variables) with the exception of a tendency for a higher peak respiratory exchange ratio in the impaired fitness group $(P=0.086)$. Survivors with impaired fitness also had significantly lower $\mathrm{VO}_{2}$ at the AT $(P=0.025)$, which on 
Table 2 Exercise capacity and cardiopulmonary parameters

\begin{tabular}{|c|c|c|c|}
\hline & Normal Fitness & Impaired Fitness & $P$ \\
\hline \multicolumn{4}{|l|}{ Cardiopulmonary Exercise Testing } \\
\hline$\%$ Predicted $H R_{\max }(\%)$ & $99 \pm 7$ & $98 \pm 5$ & 0.65 \\
\hline Respiratory exchange ratio & $1.20 \pm 0.07$ & $1.33 \pm 0.20$ & 0.086 \\
\hline Peak Workload (watts) & $234 \pm 89$ & $156 \pm 85$ & 0.089 \\
\hline Exercise Time (mins) & $9.0(6.0,12.0)$ & $8.5(5.3-11.8)$ & 0.22 \\
\hline Peak $\mathrm{VO}_{2}(\mathrm{~L} / \mathrm{min})$ & $2.5 \pm 1.1$ & $1.5 \pm 0.7$ & 0.018 \\
\hline Peak $\mathrm{VO}_{2}(\mathrm{~mL} / \mathrm{kg} / \mathrm{min})$ & $40.8 \pm 10.0$ & $27.3 \pm 7.4$ & 0.003 \\
\hline$\%$ Predicted Peak $\mathrm{VO}_{2}(\%)$ & $97 \pm 14$ & $70 \pm 16$ & 0.001 \\
\hline $\mathrm{VO}_{2}$ at $\mathrm{AT}(\mathrm{L} / \mathrm{min})$ & $1.7 \pm 0.7$ & $1.1 \pm 0.4$ & 0.025 \\
\hline$\%$ Predicted Peak $\mathrm{VO}_{2}$ at AT (\%) & $66 \pm 12$ & $50 \pm 14$ & 0.02 \\
\hline
\end{tabular}

Values are mean \pm SD or median (interquartile range). Abbreviations: AT Anaerobic threshold, HR Heart rate, RER Respiratory exchange ratio

average, occurred at $50 \%$ of their predicted peak $\mathrm{VO}_{2}$, compared to $66 \%$ of predicted peak $\mathrm{VO}_{2}$ in the normal fitness group $(P=0.02)$.

\section{Resting cardiac function}

Results for resting echocardiographic and CMR measures of cardiac function for both groups are summarized in Table 3. Echocardiographic and CMR-derived GLS could not be obtained in one participant due to sub-optimal image quality. Despite marked differences in exercise capacity, there were no significant differences in any measures obtained from resting echocardiography, with both groups showing similar resting GLS (Supplementary Fig. 1). Three participants (38\%) with normal fitness and five participants (42\%) with impaired fitness were classified with reduced LVEF ( $\leq 53 \%)$, whilst two with normal fitness (25\%) and three with impaired fitness (25\%) were classified with reduced LV FS $(<28 \%)$. There were no differences in the proportion of each group with impaired resting LVEF or FS ( $P>0.05$ for both measures). Resting CMR-derived LVEF was similar between groups, whilst RVEF was significantly higher $(P=0.006)$ and SVI tended to be lower $(P=0.09)$ in the impaired fitness group. In contrast, there were no differences in resting CI (Fig. 1), nor CMRderived GLS (Table 3; also see Additional file 1, Supplementary Fig. 1 which illustrates the individual values for GLS for both groups).

\section{Cardiac reserve}

Results related to the central hemodynamic response and contractile reserve are presented in Fig. 1a-c and Fig. 2a-b, respectively. Survivors with impaired fitness had a reduced increase in cardiac index (CI) from rest to peak exercise (Fig. 1a; group-by-exercise response interaction $P=0.001$ ), such that $\mathrm{CI}$ at peak exercise was $24 \%$ lower than survivors with normal fitness (Table 3: $6.8 \pm$ 1.2 vs $\left.8.9 \pm 1.6 \mathrm{~L} / \mathrm{min} / \mathrm{m}^{2}, P<0.01\right)$. The impaired CI response appears to be due to differences in SVI reserve
(Fig. 1b) as the heart rate response was similar (Fig. 1c). Specifically, survivors with impaired fitness had a markedly attenuated SVI response to exercise (group-by-exercise response interaction $P=0.001$ ) and a reduced peak SVI (Table 3: peak exercise SVI 27\% lower, $P<0.01$ ). Those with normal fitness showed a significantly higher

Table 3 Resting and peak cardiac function

\begin{tabular}{|c|c|c|c|}
\hline & Normal Fitness & Impaired Fitness & $P$ \\
\hline \multicolumn{4}{|c|}{ Resting Echocardiography } \\
\hline LVEF, \% & $55.1 \pm 3.6$ & $55.3 \pm 8.6$ & 0.97 \\
\hline LV FS, \% & $32.9 \pm 5.4$ & $30.5 \pm 5.0$ & 0.32 \\
\hline GLS, \% & $-18.6 \pm 2.4$ & $-17.8 \pm 2.1^{\mathrm{a}}$ & 0.46 \\
\hline E/A & $1.87(1.09,2.65)$ & $1.79(0.77,2.81)$ & 0.79 \\
\hline$E / e^{\prime}$ & $6.0(4.4,7.6)$ & $6.8(5.1,8.5)$ & 0.26 \\
\hline DT, $\mathrm{cm} / \mathrm{s}$ & $183 \pm 23$ & $176 \pm 64$ & 0.64 \\
\hline \multicolumn{4}{|l|}{ Resting CMR } \\
\hline GLS, \% & $-15.3 \pm 2.3$ & $-14.9 \pm 2.0^{b}$ & 0.69 \\
\hline LVEF, \% & $53.8 \pm 3.8$ & $52.6 \pm 6.6$ & 0.66 \\
\hline RVEF, \% & $49.4 \pm 4.0$ & $55.6 \pm 4.8$ & 0.006 \\
\hline $\mathrm{SVI}, \mathrm{mL} / \mathrm{m}^{2}$ & $51 \pm 11$ & $43 \pm 9$ & 0.09 \\
\hline $\mathrm{Cl}, \mathrm{L} / \mathrm{min} / \mathrm{m}^{2}$ & $4.0 \pm 1.0$ & $3.6 \pm 0.6$ & 0.29 \\
\hline \multicolumn{4}{|l|}{ Exercise CMR } \\
\hline $\mathrm{LVEF}_{\text {peak, }} \%$ & $65.0 \pm 3.2$ & $60.0 \pm 7.8$ & 0.07 \\
\hline RVEF $_{\text {peak, }} \%$ & $62.8 \pm 5.1$ & $63.9 \pm 6.3$ & 0.67 \\
\hline$S V I_{\text {peak }}, \mathrm{mL} / \mathrm{m}^{2}$ & $60 \pm 15$ & $44 \pm 9$ & 0.007 \\
\hline $\mathrm{Cl}_{\text {peak, }} \mathrm{L} / \mathrm{min} / \mathrm{m}^{2}$ & $9.0 \pm 1.6$ & $6.8 \pm 1.2$ & 0.003 \\
\hline$a-\mathrm{vO}_{2}$ diffe & $16.3 \pm 3.3$ & $12.9 \pm 2.5$ & 0.016 \\
\hline
\end{tabular}

Values are means \pm SD or median (interquartile range). LV Left ventricular, LVEF Left ventricular ejection fraction, FS Fractional shortening, GLS Global longitudinal strain, E/A Ratio of peak early-to-late mitral inflow velocities, E/e Ratio of peak mitral inflow to peak early mitral annular velocity, $D T$ Deceleration time, CMR Cardiovascular magnetic resonance imaging, RVEF Right ventricular ejection fraction, SVI Stroke volume index, $\mathrm{Cl}$ Cardiac index, $a$ $\mathrm{vO}_{2}$ diff Arterio-venous oxygen difference. ${ }^{\mathrm{a}} n=7 ;{ }^{\mathrm{b}} n=11$; $^{\mathrm{c}}$ calculated using peak $\mathrm{VO}_{2}$ from cardiopulmonary exercise test and peak cardiac output from exercise $C M R$ 


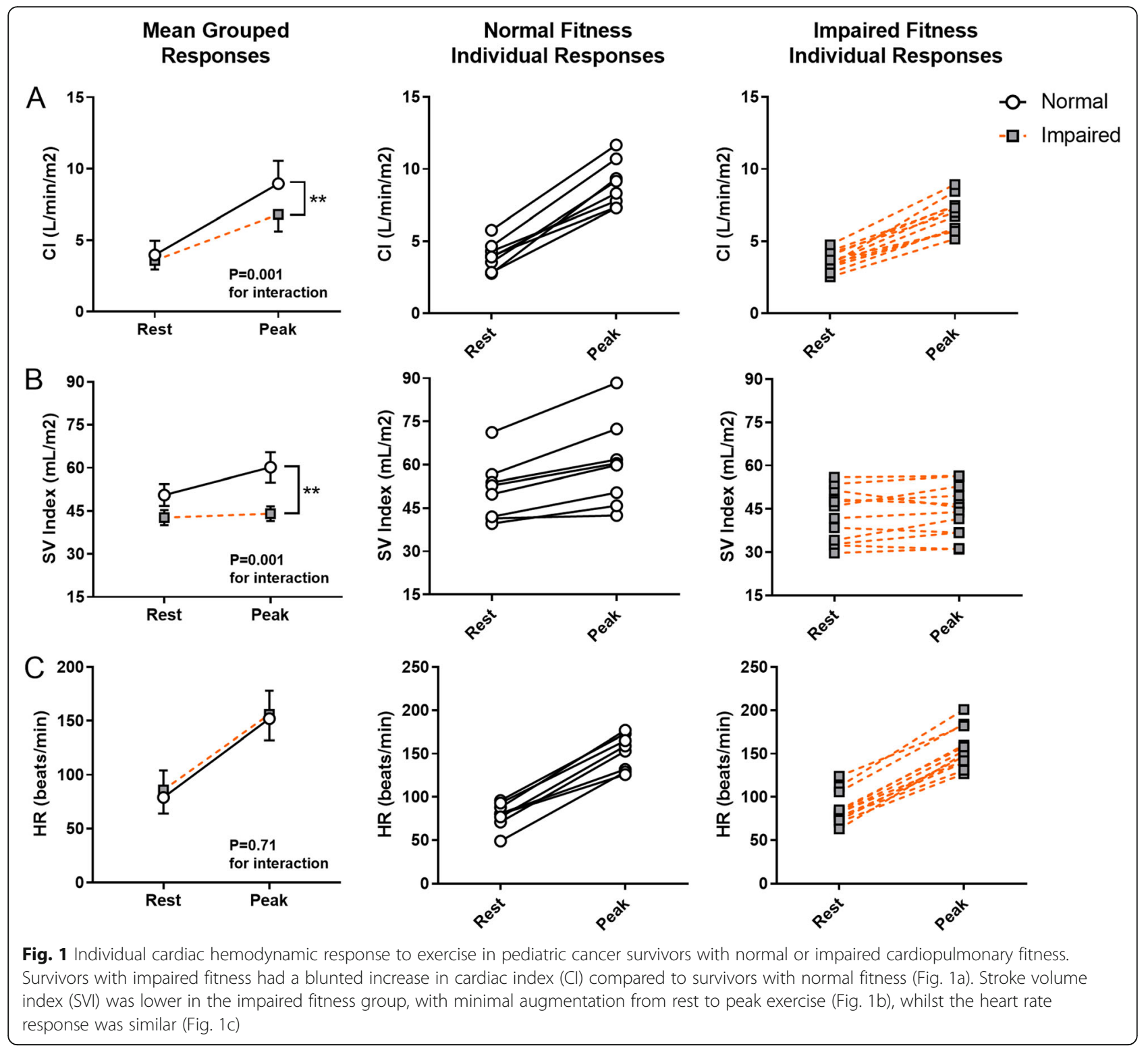

augmentation of both LVEF and RVEF (Fig. 2a and b, group-by-exercise response interaction $P=0.011$ and $P=0.002$, respectively). The difference in LVEF response appears to be due to a higher peak LVEF in the normal fitness group $(P=0.069)$ as the resting LVEF was similar $(P=0.66)$. In contrast, the difference in RVEF response is largely due to a lower resting RVEF in the normal fitness group $(P=0.006)$, and a similar RVEF between groups at peak exercise $(P=0.68)$. A video providing an example of the biventricular exercise response assessed by exercise CMR in a survivor with normal fitness and a survivor with impaired fitness is shown in Additional file 1. Those with impaired fitness also had significantly lower $\mathrm{a}-\mathrm{vO}_{2}$ difference at peak exercise $(12.9 \pm 2.5$ vs $16.3 \pm 3.3, P=0.016)$.
Associations between exercise capacity with central and peripheral function

Associations between peak $\mathrm{VO}_{2}$ and measures of cardiac and peripheral function are displayed in Table 4. There were strong associations between peak $\mathrm{VO}_{2}$ and measures of cardiac reserve (peak CI and peak SVI) and peripheral oxygen extraction. In contrast, there were no significant associations between peak $\mathrm{VO}_{2}$ and resting echocardiographic and CMR indices of cardiotoxicity (resting LV FS, LVEF and GLS).

\section{Discussion}

The main finding from this study was that the classification of pediatric cancer survivors into normal or reduced cardiopulmonary fitness identifies two distinct 


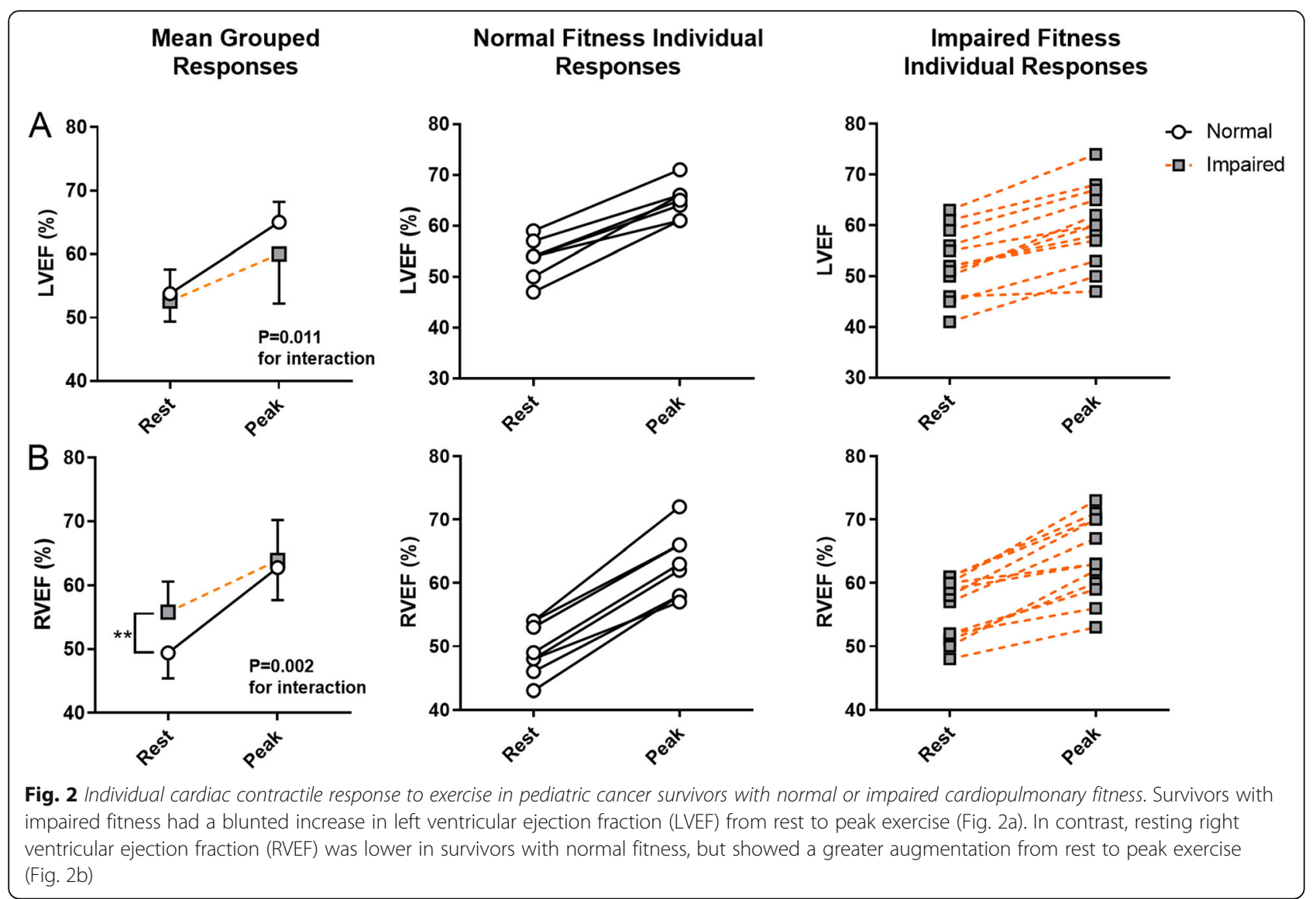

phenotypes. Specifically, those with normal fitness have a significant augmentation of $\mathrm{CI}$, which occurs due to a significant increase in both heart rate and SVI. In contrast, survivors with impaired fitness have a blunted increase in CI that is primarily driven by increases in heart rate and minimal change in SVI. These differences in fitness and exercise cardiac reserve exist despite no significant differences between groups in measures of cardiac function currently used to quantify heart failure risk and guide clinical decision making.

There is growing awareness of the limitations for the current approach of cardiotoxicity screening in pediatric cancer survivors $[6,8]$. Current guidelines are centered on detecting $>10 \%$ reductions in resting fractional shortening or LVEF $[6,8]$, however there is little evidence to show that this approach reliably predicts subsequent cardiovascular morbidity and mortality [6]. Furthermore, reductions in these measures that remain within the normal range show little correlation with exercise capacity or quality of life [23]. We have investigated an alternative approach that focuses on peak $\mathrm{VO}_{2}$, a holistic measure of cardiovascular reserve that has been shown to correlate with heart failure incidence $[24,25]$, prognosis [26, 27] and quality of life [28] among healthy and clinical cohorts. On average, our cohort had a peak $\mathrm{VO}_{2}$ that was $19 \%$ below predicted values for age, gender and height. This is consistent with a number of other studies documenting pediatric cancer survivors previously treated with anthracyclines and/or chest radiation having a peak $\mathrm{VO}_{2}$ that is $8-20 \%$ below predicted values or matched control subjects $[14,29,30]$. However, by dividing the spectrum of exercise responses into a binary

Table 4 Correlations between peak $\mathrm{VO}_{2}$ with cardiac and peripheral measures

\begin{tabular}{lll}
\hline Measure & $\mathrm{R}^{2}$ & $\boldsymbol{P}$-value \\
\hline Peak exercise measures & & \\
Peak Cl & 0.59 & $<0.001$ \\
Peak SVI & 0.67 & $<0.001$ \\
a-vO ${ }_{2}$ diff & 0.71 & $<0.001$ \\
Resting measures & & \\
LVEF & 0.01 & 0.55 \\
LV FS & 0.01 & 0.75 \\
Echocardiographic GLS & -0.10 & 0.19 \\
CMR GLS & -0.03 & 0.49 \\
\hline
\end{tabular}

Abbreviations: $\mathrm{Cl}$ Cardiac index, $a-\mathrm{vO}_{2}$ diff Arterio-venous oxygen difference, LVEF Left ventricular ejection fraction, LVFS Left ventricular fractional shortening, GLS Global longitudinal strain, CMR Cardiovascular magnetic resonance 
construct, we have identified a group with marked exercise impairment who have demonstrable abnormalities in cardiac function inducible during exercise stress. The reduction in exercise capacity and cardiac function is likely to be a persistent effect of treatment, as crosssectional studies have reported that between 30 and $79 \%$ of long-term survivors measured $>5$ years posttreatment have impaired peak $\mathrm{VO}_{2}$ [30-32]. Notably, evidence from large prospective studies in non-cancer populations has shown that incident heart failure risk increased by $16 \%$ for each $3.5 \mathrm{~mL} / \mathrm{kg} / \mathrm{min}$ decrement in peak $\mathrm{VO}_{2}$ [24, 25]. Given the average peak $\mathrm{VO}_{2}$ for the impaired fitness group was $12.1 \mathrm{~mL} / \mathrm{kg} / \mathrm{min}$ below predicted, we would infer this group is at substantially greater risk of development of heart failure symptoms over time than the normal fitness group, who on average, achieved almost $100 \%$ of predicted peak $\mathrm{VO}_{2}$. This ensues despite no significant differences between our two study groups in resting echocardiographic measures of LV FS and LVEF, nor even in more novel echocardiographic and CMR-derived measures of GLS. This highlights that the current standard of care approach to quantifying cardiac function is insensitive to clinically important differences in peak $\mathrm{VO}_{2}$ and heart failure risk.

Given that heart failure is typified by an inability of cardiac function to meet metabolic demand, we hypothesized that assessing cardiac function during periods of increased metabolic demand (such as exercise) should be more sensitive to heart failure risk than the assessment of cardiac function at rest when metabolic demands are low. The inclusion of novel exercise cardiac imaging has allowed us to demonstrate cardiac reserve is significantly reduced in pediatric cancer survivors with reduced peak $\mathrm{VO}_{2}$. Specifically, we found that survivors with reduced peak $\mathrm{VO}_{2}$ showed a blunted increase in LVEF and minimal augmentation of SVI during exercise, resulting in a blunted increase in exercise $\mathrm{CI}$ such that peak exercise CI was $24 \%$ lower than survivors with normal fitness. Two cross-sectional reports in pediatric cancer survivors have reported similar results, with survivors demonstrating lower exercise SVI and CI than age- and gender-matched controls [33, 34]. However, our study is the first in pediatric cancer survivors to document this relationship with impaired peak $\mathrm{VO}_{2}$. A handful of cross-sectional studies have investigated cardiac impairment in long-term pediatric cancer survivors with normal or reduced peak $\mathrm{VO}_{2}[29,30,35]$. However results from these studies have been inconsistent, which may relate to the assessment of cardiac function in the resting state. This is supported by findings from our study, in which there was no significant difference in resting LV FS, LVEF or GLS between our two study groups, nor did these measures correlate with peak $\mathrm{VO}_{2}$. The largest of these previous studies [35] demonstrated that impaired peak $\mathrm{VO}_{2}$ was associated with reduced resting GLS, but not resting LVEF in 1041 long-term (> 10 years) pediatric cancer survivors, supporting the notion that exercise intolerance is a maker of cardiac dysfunction in cancer survivors. However, results from our study suggest that impairments in fitness and cardiac reserve may precede impairment in GLS. The fact that RVEF was paradoxically lower at rest in fitter survivors is consistent with work in athletic populations in which lower resting RVEF is associated with greater fitness [36]. In the current population, this paradox could create diagnostic confusion and again emphasizes the potential errors that are created by reliance on resting measures and the clarity provided by exercise. Taken together, our results highlight the importance of exercise-based imaging approaches to unmask cardiac dysfunction in cancer survivors.

The relationship between exercise capacity and heart failure risk in cancer survivors is a matter of ongoing investigation. Pandey et al. [37] reported that the greatest long-term risk of heart failure was observed in middleaged adults with reduced exercise capacity and it is reasonable to speculate that those with markedly reduced exercise capacity as an adolescent would be at even greater risk. Peak $\mathrm{VO}_{2}$ is determined by a combination of cardiac (central) and non-cardiac (peripheral) factors, and Houstis et al. [38] have shown 97\% of heart failure patients referred for exercise testing had significant limitation in both. Furthermore, it was estimated that complete reversal of cardiac impairment would only improve peak $\mathrm{VO}_{2}$ by $7 \%$, but complete improvement in peripheral oxygen extraction would improve peak $\mathrm{VO}_{2}$ by $27 \%$ [38]. This draws interesting parallels to the current study, where we observed survivors with reduced peak $\mathrm{VO}_{2}$ had significant limitation in both cardiac function and peripheral oxygen extraction. Previous studies $[39,40]$ have documented impairment in peripheral oxygen extraction in breast cancer patients receiving anthracycline- and/or taxane-based chemotherapy, however the existence of peripheral impairment in pediatric cancer survivors is a new and important finding. Given the majority of therapeutic strategies for heart failure prevention in cancer survivors are 'cardiac focused', these results highlight the need to investigate adjunctive therapies such as exercise training, that can address peripheral limitations [40]. This also highlights the value of an integrative diagnostic measure such as peak $\mathrm{VO}_{2}$, which is able to capture the central and peripheral defects that can contribute to exercise intolerance.

The primary limitation to this study is the modestly sized, heterogenous cohort. Whilst the modest cohort size increases the likelihood of a type I error, the alternative view that the differences noted in this study could represent a large effect size, should not be discounted. Indeed, 
the findings are not driven by outliers (Figs. 1 and 2), and the ability of our exercise measures to detect significant differences in cardiac function in such a small sample supports the higher sensitivity associated with exercise-based assessment of cardiac function. The small sample size also limits our ability to understand the relationships between host and treatment factors which are associated with reduced exercise capacity. Whilst participants had undergone varying degrees of cancer treatment, this represents the variety of real-world clinical contexts by which cancer survivors present with exertional intolerance. Additionally, the prognostic significance of the relationship between reduced peak $\mathrm{VO}_{2}$ and cardiac reserve impairment in pediatric cancer survivors is unclear. Whilst we may speculate by drawing on parallels from other forms of heart failure, our findings require further validation to understand the prospective relationship between reduced peak $\mathrm{VO}_{2}$ and clinical endpoints such as heart failure incidence and prognosis.

\section{Conclusions}

Pediatric cancer survivors with impaired peak $\mathrm{VO}_{2}$ demonstrate reduced cardiac functional reserve, despite having similar resting cardiac function to survivors with normal peak $\mathrm{VO}_{2}$. This suggests that measurement of peak $\mathrm{VO}_{2}$ or cardiac functional reserve may provide additional benefit in early identification of survivors at increased risk of cardiac dysfunction and heart failure.

\section{Supplementary information}

Supplementary information accompanies this paper at https://doi.org/10. 1186/s12968-020-00658-4.

\footnotetext{
Additional file 1 Example of a pediatric cancer survivor with good and poor biventricular haemodynamic and contractile reserve assessed by exercise cardiac magnetic resonance imaging. The survivor with poor cardiac reserve showed little augmentation in stroke volume (Rest: 42 $\mathrm{mL} / \mathrm{m}^{2}$ vs Peak: $44 \mathrm{~mL} / \mathrm{m}^{2}$ ), LVEF (Rest: $53 \%$ vs Peak: $57 \%$ ) or RVEF (Rest: $52 \%$ vs Peak: $56 \%$ ), and had a peak $\mathrm{VO}_{2} 73 \%$ of predicted. In contrast, the survivor with good cardiac reserve showed significant augmentation of stroke volume (Rest: $42 \mathrm{~mL} / \mathrm{m}^{2}$ vs Peak: $50 \mathrm{~mL} / \mathrm{m}^{2}$ ), LVEF (Rest: $55 \%$ vs Peak: 66\%) and RVEF (Rest: $54 \%$ vs Peak: $66 \%$ ), and had a peak $\mathrm{VO}_{2}$ that was $99 \%$ of predicted.
}

Additional file 1 Figure S1. Individual resting global longitudinal strain assessed by cardiac magnetic resonance imaging and speckle tracking echocardiography in pediatric cancer survivors with normal or impaired cardiopulmonary fitness. Individual values (with overlay of mean and 95\% Cl) demonstrating that survivors with normal and impaired fitness had comparable resting global longitudinal strain (GLS) assessed by cardiac magnetic resonance imaging (CMR) and speckle-tracking echocardiography (STE).

\section{Abbreviations}

2D: Two-dimensional; a-vO $\mathrm{V}_{2}$ diff: Arterio-venous oxygen difference; BMI: Body mass index; Cl: Cardiac index; CMR: Cardiovascular magnetic resonance; CPET: Cardiopulmonary exercise test; ECG: Electrocardiogram; exCMR: Exercise cardiac magnetic resonance imaging; FS: Fractional shortening; GLS: Global longitudinal strain; LV: Left ventricle/left ventricular; LVEF: Left-ventricular ejection fraction; Peak $\mathrm{VO}_{2}$ : Peak oxygen consumption;
RER: Respiratory exchange ratio; RV: Right ventricle/right ventricular; RVEF: Right-ventricular ejection fraction; SVI: Stroke volume index

\section{Acknowledgements}

Not applicable.

\section{Authors' contributions}

All authors made significant contributions to the published study. ALG, BC, $P C, R C$ and DAE were responsible for the concept and design of the study design and procedures. SF, BC, EJH, HD, CT and $\mathrm{KJ}$ were responsible for subject recruitment and data collection. SF, BC, PC and ALG were responsible for image analysis. SF and $R C$ conducted the statistical analysis. All authors contributed to the writing and critical review of this manuscript and have approved the final version of this manuscript.

\section{Funding}

This research was funded, in part, from A La Gerche's National Heart Foundation Future Leader Fellowship (App 102021 "Utility of novel exercise imaging to identify sub-clinical cardiovascular disease"). DAE and RC are supported by the Royal Children's Hospital Foundation, Childrens Cancer Foundation and the Kids Cancer Project. RC is a recipient of an MCRI ClinicianScientist Fellowship. SF is supported by an Australian Government Research Training Program Scholarship (RTP 4635089552).

\section{Availability of data and materials}

The datasets used and/or analysed during the current study are available from the corresponding author on reasonable request.

Ethics approval and consent to participate

Participants provided informed written consent prior to participation in the study and additional exercise assessments. This study was approved by the ethics committee of the Melbourne Royal Children's Hospital (HREC 35102D) and the Alfred Hospital (HREC 00315) and was conducted in accordance with the Declaration of Helsinki guidelines.

\section{Consent for publication}

Not applicable.

\section{Competing interests}

The authors declare that they have no competing interests.

\section{Author details}

${ }^{1}$ Department of Sports Cardiology, Baker Heart and Diabetes Institute, 75 Commercial Rd, Melbourne, VIC 3004, Australia. ${ }^{2}$ Institute for Physical Activity and Nutrition, Deakin University, Geelong, VIC, Australia. ${ }^{3}$ Cardiology Department, St Vincent's Hospital Melbourne, Melbourne, VIC, Australia. ${ }^{4}$ Children's Cancer Centre, The Royal Children's Hospital, Melbourne, VIC, Australia. ${ }^{5}$ Department of Cardiovascular Sciences, KU Leuven, Leuven, Belgium. 'Murdoch Children's Research Institute, The Royal Children's Hospital, Melbourne, VIC, Australia. ${ }^{7}$ Department of Paediatrics, University of Melbourne, Parkville, VIC, Australia. ${ }^{8}$ Medicine, Dentistry and Health Sciences, University of Melbourne, Parkville, VIC, Australia.

Received: 12 January 2020 Accepted: 28 July 2020

Published online: 07 September 2020

\section{References}

1. Siegel RL, Miller KD, Jemal A. Cancer statistics, 2016. CA Cancer J Clin. 2016; $66: 7-30$.

2. Oeffinger KC, Mertens AC, Sklar CA, et al. Chronic health conditions in adult survivors of childhood cancer. N Engl J Med. 2006;355:1572-82.

3. Tukenova M, Guibout C, Oberlin O, et al. Role of cancer treatment in longterm overall and cardiovascular mortality after childhood cancer. J Clin Oncol. 2010:28:1308-15.

4. Armstrong GT, Liu Q, Yasui Y, et al. Late mortality among 5-year survivors of childhood cancer: a summary from the childhood cancer survivor study. J Clin Oncol. 2009;27:2328

5. Haddy N, Diallo S, El-Fayech C, et al. Cardiac diseases following childhood cancer treatment: cohort study. Circulation. 2016;133:31-8.

6. Lipshultz SE, Adams MJ, Colan SD, et al. Long-term cardiovascular toxicity in children, adolescents, and young adults who receive cancer therapy: 
pathophysiology, course, monitoring, management, prevention, and research directions: a scientific statement from the American Heart Association. Circulation. 2013;128:1927-95.

7. Nathan P, Greenberg M, Ness K, et al. Risk-based care in survivors of childhood cancer: a report from the childhood cancer survivor study (CCSS). J Clin Oncol. 2007;25:6502.

8. Chow EJ, Leger KJ, Bhatt NS, et al. Paediatric cardio-oncology: epidemiology, screening, prevention, and treatment. Cardiovasc Res. 2019;115:922-34. https://doi.org/10.1093/cvr/cvz031.

9. Ewer MS, Lenihan DJ. Left ventricular ejection fraction and cardiotoxicity: is our ear really to the ground? J Clin Oncol. 2008;26:1201-3. https://doi.org/ 10.1200/JCO.2007.14.8742.

10. Thavendiranathan P, Grant AD, Negishi T, et al. Reproducibility of echocardiographic techniques for sequential assessment of left ventricular ejection fraction and volumes. J Am Coll Cardiol. 2013;61:77-84. https://doi. org/10.1016/j.jacc.2012.09.035.

11. Balady GJ, Arena R, Sietsema K, et al. Clinician's guide to cardiopulmonary exercise testing in adults. Circulation. 2010;122:191. https://doi.org/10.1161/ CIR.0b013e3181e52e69.

12. Corrà U, Piepoli MF, Adamopoulos S, et al. Cardiopulmonary exercise testing in systolic heart failure in 2014: the evolving prognostic role. Eur J Heart Fail. 2014;16:929-41.

13. La Gerche A, Claessen G, Van de Bruaene A, et al. Cardiac MRI: a new gold standard for ventricular volume quantification during high-intensity exercise. Circ Cardiovasc Imaging. 2013;6:329-38Comparative Study Research Support, Non-U.S. Gov't Validation Studies 2012/12/22. https://doi. org/10.1161/CIRCIMAGING.112.980037.

14. De Caro E, Smeraldi A, Trocchio G, et al. Subclinical cardiac dysfunction and exercise performance in childhood cancer survivors. Pediatr Blood Cancer. 2011:56:122-6

15. Cifra B, Chen CK, Fan C-PS, et al. Dynamic myocardial response to exercise in childhood cancer survivors treated with anthracyclines. J Am Soc Echocardiogr. 2018;31:933-42. https://doi.org/10.1016/j.echo.2018.02.003.

16. Cooper DM, Weiler-Ravell D. Gas exchange response to exercise in children. Am Rev Respir Dis. 1984;129:S47-8.

17. de Souza e Silva CG, Kaminsky LA, Arena R, et al. A reference equation for maximal aerobic power for treadmill and cycle ergometer exercise testing: analysis from the FRIEND registry. Eur J Prev Cardiol. 2018;25:742-50.

18. American Thoracic Society. ATS/ACCP statement on cardiopulmonary exercise testing. Am J Respir Crit Care Med. 2003;167:211-77. https://doi. org/10.1164/rccm.167.2.211.

19. Schneider DA, Phillips SE, Stoffolano S. The simplified V-slope method of detecting the gas exchange threshold. Med Sci Sports Exerc. 1993;25:11804 1993/10/01.

20. Lang RM, Badano LP, Mor-Avi V, et al. Recommendations for cardiac chamber quantification by echocardiography in adults: an update from the American Society of Echocardiography and the European Association of Cardiovascular Imaging. J Am Soc Echocardiogr. 2015;28:1-39e14. https:// doi.org/10.1016/j.echo.2014.10.003.

21. Lang RM, Badano LP, Mor-Avi V, et al. Recommendations for cardiac chamber quantification by echocardiography in adults: an update from the American Society of Echocardiography and the European Association of Cardiovascular Imaging. Eur Heart J Cardiovasc Imaging. 2015;16:233702015/02/26. https://doi.org/10.1093/ehjci/jev014.

22. McMichael J, Sharpey-Schafer EP. Cardiac output in man by a direct Fick method: effects of posture, venous pressure change, atropine, and adrenaline. Br Heart J. 1944;6:33-40 1944/01/01.

23. Franciosa JA, Park M, Barry Levine T. Lack of correlation between exercise capacity and indexes of resting left ventricular performance in heart failure. Am J Cardiol. 1981;47:33-9. https://doi.org/10.1016/0002-9149(81)90286-1.

24. Khan H, Kunutsor S, Rauramaa R, et al. Cardiorespiratory fitness and risk of heart failure: a population-based follow-up study. Eur J Heart Fail. 2014;16: 180-8.

25. Kupsky DF, Ahmed AM, Sakr S, et al. Cardiorespiratory fitness and incident heart failure: the Henry ford Exerclse testing (FIT) project. Am Heart J. 2017 185:35-42.

26. Kodama S, Saito K, Tanaka S, et al. Cardiorespiratory fitness as a quantitative predictor of all-cause mortality and cardiovascular events in healthy men and women: a meta-analysis. JAMA. 2009;301:2024-35.

27. Keteyian SJ, Patel M, Kraus WE, et al. Variables measured during cardiopulmonary exercise testing as predictors of mortality in chronic systolic heart failure. J Am Coll Cardiol. 2016;67:780-9. https://doi.org/10. 1016/j.jacc.2015.11.050

28. Adams MJ, Ng AK, Mauch P, et al. Peak oxygen consumption in Hodgkin's lymphoma survivors treated with mediastinal radiotherapy as a predictor of quality of life 5years later. Prog Pediatr Cardiol. 2015;39:93-8. https://doi.org/ 10.1016/j.ppedcard.2015.10.006.

29. Miller AM, Lopez-Mitnik G, Somarriba G, et al. Exercise capacity in long-term survivors of pediatric cancer: an analysis from the cardiac risk factors in childhood cancer survivors study. Pediatr Blood Cancer. 2013;60:663-8.

30. Christiansen JR, Kanellopoulos A, Lund MB, et al. Impaired exercise capacity and left ventricular function in long-term adult survivors of childhood acute lymphoblastic leukemia. Pediatr Blood Cancer. 2015;62:1437-43. https://doi. org/10.1002/pbc.25492.

31. Tonorezos ES, Snell PG, Moskowitz CS, et al. Reduced cardiorespiratory fitness in adult survivors of childhood acute lymphoblastic leukemia. Pediatr Blood Cancer. 2013;60:1358-642013/02/15. https://doi.org/10.1002/pbc.24492.

32. Adams MJ, Lipsitz SR, Colan SD, et al. Cardiovascular status in long-term survivors of Hodgkin's disease treated with chest radiotherapy. J Clin Oncol. 2004;22:3139-48.

33. Johnson D, Perrault $\mathrm{H}$, Fournier A, et al. Cardiovascular responses to dynamic submaximal exercise in children previously treated with anthracycline. Am Heart J. 1997;133:169-73DOI. https://doi.org/10.1016/ S0002-8703(97)70205-9.

34. De Souza AM, Potts JE, Potts MT, et al. A stress echocardiography study of cardiac function during progressive exercise in pediatric oncology patients treated with anthracyclines. Pediatr Blood Cancer. 2007:49:56-64.

35. Ness KK, Plana JC, Joshi VM, et al. Exercise intolerance, mortality, and organ system impairment in adult survivors of childhood cancer. J Clin Oncol. 2019;38(1):JCO. 19.01661.

36. La Gerche A, Burns AT, Taylor AJ, et al. Maximal oxygen consumption is best predicted by measures of cardiac size rather than function in healthy adults. Eur J Appl Physiol. 2012;112:2139-47.

37. Pandey A, Cornwell WK, Willis B, et al. Body mass index and cardiorespiratory fitness in mid-life and risk of heart failure hospitalization in older age: findings from the Cooper Center longitudinal study. JACC: Heart Failure. 2017:5:367-74.

38. Houstis NE, Eisman AS, Pappagianopoulos PP, et al. Exercise intolerance in heart failure with preserved ejection fraction: diagnosing and ranking its causes using personalized O2 pathway analysis. Circulation. 2018;137:148-61.

39. Howden EJ, Bigaran A, Beaudry R, et al. Exercise as a diagnostic and therapeutic tool for the prevention of cardiovascular dysfunction in breast cancer patients. Eur J Prev Cardiol. 2018:0:1-11 30 Oct 2018.

40. Mijwel S, Cardinale DA, Norrbom J, et al. Exercise training during chemotherapy preserves skeletal muscle fiber area, capillarization, and mitochondrial content in patients with breast cancer. FASEB J. 2018;32: 5495-505.

\section{Publisher's Note}

Springer Nature remains neutral with regard to jurisdictional claims in published maps and institutional affiliations.

Ready to submit your research? Choose BMC and benefit from:

- fast, convenient online submission

- thorough peer review by experienced researchers in your field

- rapid publication on acceptance

- support for research data, including large and complex data types

- gold Open Access which fosters wider collaboration and increased citations

- maximum visibility for your research: over $100 \mathrm{M}$ website views per year

At $\mathrm{BMC}$, research is always in progress.

Learn more biomedcentral.com/submissions 